\title{
Obstetric recto-vaginal fistula: What technique to use?*
}

\author{
Sanaa Errarhay ${ }^{\#}$, Samia Mahmoud, Najia Hmidani, Hanane Saadi, Chahrazed Bouchikhi, \\ Abdelaziz Banani \\ Department of Gynecology and Obstetrics, CHU Hassan II, Fez, Morroco \\ Email: ${ }^{\text {suine_err@yahoo.fr }}$
}

Received 25 May 2013; revised 22 June 2013; accepted 29 June 2013

Copyright (C) 2013 Sanaa Errarhay et al. This is an open access article distributed under the Creative Commons Attribution License, which permits unrestricted use, distribution, and reproduction in any medium, provided the original work is properly cited.

\begin{abstract}
Objective: The recto-vaginal fistula is an abnormal communication between the previous wall of the rectum and the posterior face of the vagina through the recto-vaginal septum. They are often due to the obstetric traumas and they can be of post-surgical origin. Methods: We report five cases of patients with obstetric recto-vaginal fistula and all of the patients have surgical intervention with a resection of the fistulous route and rectal wall. Results: The patient did not present any recidivating and the evolution is satisfactory without complications or recidivating. Conclusion: The treatment of recto-vaginal fistula is often difficult exposed to recurrence; and the therapeutic indications must be adapted to every type of fistula by taking into account its size, its location and its etiology.
\end{abstract}

Keywords: Recto-Vaginal; Obstetric Trauma; Pelvic Trauma; Treatment

\section{INTRODUCTION}

The recto-vaginal fistula is an abnormal communication between the rectum and the vagina through the rectovaginal septum. They can be post-obstetric, post-surgical or after non-operated accident. These fistulas are translated by several clinical boards starting from a simple discomfort to plentiful losses of stool. The treatment is simple and will be more effective if done early after the accident.

Through 5 cases operated in our department of gynecology and obstetrics, we are going to make a review article on the surgical difficulties of this type of pathology, since there exist several surgical techniques. We shall insist on the technique which we use in the department, namely the resection of the fistulous route and the

\footnotetext{
*Conflict of interest: The authors declare that they have no conflict of interest.

\#Corresponding author.
}

fibrosis zone then the suture of the rectum and the vagina.

\section{OBSERVATION NO. 1}

It is about a multifarious 55-year-old patient, without considerable pathological histories. All the deliveries took place at home with non-specified born weights. The patient reports since her marriage, 40 years ago, the stemming of the stools by the vagina. The gynecological examination objectified, by the speculum and in the rectal touch combined to the vaginal touch, a solution of continuity measuring $5 \mathrm{~cm}$ at the level of the posterior vaginal wall with the stemming of the stool through an opening situated in $1 \mathrm{~cm}$ of the anal margin.

A rectoscopy showed a $4 \mathrm{~cm}$ wide recto-vaginal fistula with normal bank and a non-inflammatory mucous membrane.

The patient benefited, after a pre-operative preparation with a rectal injection, from a resection of the fistulous route and the fibrosis zone with suture of the rectum and the vagina. The operating room is sent for histo-pathologic study which did not objectify specific or tumoral lesions.

After the surgical operation, the patient is kept under parenteral alimentation during a week and under antibiotic treatment with protected amoxicilline $3 \mathrm{~g}$ a day and metronidazol $1 \mathrm{~g}$ a day with good clinical evolution.

\section{OBSERVATION NO. 2}

It is about a primigravid and primiparous 25-year-old patient, without considerable pathological histories having given birth, 2 years ago by the vaginal way, to a newborn child in presentation of complete breech weighing 3200 grams with episiotomy. The post-partum is marked, to 12th day, by the stemming of stools by the vagina without notion of neither genital infections with repetition nor urinary recurrent infections.

The clinical examination revealed a recto-vaginal fistula 
with $1 \mathrm{~cm}$ of the anal margin. The patient benefited from a rectoscopy which objectified a rectal mucous membrane of normal appearance while objectifying the rectovaginal fistula.

The operation was preceded by an intestinal preparation. The patient benefited from a resection of the fistulous route and from the fibrosis zone with suture of the rectum and the vagina. The patient was put under parenteral alimentation during a week and under antibiotic treatment with amoxicilline protected $3 \mathrm{~g}$ a day and metronidazol $1 \mathrm{~g}$ a day. The operating follow ups were simple with a three year retrospect.

\section{OBSERVATION NO. 3}

It is about a 23-year-old patient, without considerable pathological histories. The First vaginal delivery with episiotomy of a newborn child weighing 3200 grammes, the second vaginal delivery is realized with a male newborn child weighing 4000 grammas medically served without episiotomy nor instrumentation with the occurrence of perennial fissure: not complicated complete perineum. The immediate post-partum was marked by the emission of stools by the vagina.

The clinical in immediate post-partum examination showed a recto- vaginal fistula of $1 \mathrm{~cm}$ with stemming of stool. The patient benefited from an intestinal preparation then from a case-by-case review of the fistula, from the resection of the fistulous route then suture. The patient was put under parenteral alimentation during a week and under antibiotic treatment with amoxicilline protected 3 $\mathrm{g}$ a day and metronidazol $1 \mathrm{~g}$ a day. The operative follow ups were simple and the patient did not present any recidivating.

\section{OBSERVATION NO. 4}

It is about a primigravid and primiparous 28-year-old patient, without particular pathological histories. First childbirth is realized with episiotomy by vaginal delivery with a newborn child weighing 3800 grammas. To the third day of the post-partum, the patient noticed the stemming of stool through the vagina, but she consulted only 4 months later. The clinical examination revealed in the speculum a small cervical redness, whitish leucorrhoea with presence of a recto-vaginal fistula of $1 \mathrm{~cm}$ in $1 \mathrm{~cm}$ of the anal opening. The patient received an intestinal preparation the day before her surgical intervention then a resection of the fistulous route and the suture of the vaginal and rectal wall.

In post operative, the patient was kept under parenteral alimentation during a week and under antibiotic treatment with amoxicilline protected $3 \mathrm{~g}$ a day and metronidazol $1 \mathrm{~g}$ a day. The evolution is satisfactory without complications or recidivating.

\section{OBSERVATION NO. 5}

A 36-year-old patient, with the first non monitored pregnancy marked by a laborious childbirth, 4 years ago by below delivery with episiotomy and vacuum extractor of a female newborn child weighing $4 \mathrm{~kg} 700 \mathrm{~g}$. The patient presented in immediate post-partum the stemming of stool by the vagina but she did not consult. The 2nd pregnancy is also none monitored and the clinical examination revealed the presence of stool in the vagina with presence of a recto-vaginal fistula of $1 \mathrm{~cm}$ in $0.5 \mathrm{~cm}$ of the anal opening. A caesarean delivery is scheduled for recto-vaginal fistula. The patient benefited, 3 months later, from a cure of the recto-vaginal fistula after intestinal preparation which consisted of a perineal crossfunctional section with detachment of the recto-vaginal wall until the fistulous route and the continuation of the detachment by convoluting the fistulous route located by the stylet then the resection of the fibrosed tissue and the closure of the rectal mucous membrane after of the fistulous borders (Figure 1).

In post operative, the patient was kept under parenteral alimentation during a week and under antibiotic treatment. The evolution is satisfactory without complications or recidivating.

\section{DISCUSSION}

The recto-vaginal post-obstetric fistula is essentially the consequence of a dystocial delivery whose medical coverage was put off and a maladjusted. It is viewed in $0.12 \%$ of 20.500 deliveries in the series of Venkatesh [1]. It can occur during a spontaneous or instrumental eviction. The spontaneous fistulas represent from $80 \%$ to $90 \%$ fistulas and are secondary in a prolonged ischemic compression of the rectum by the head enclosed in the pelvis [1-4].

Three etiological factors are associated:

- The mechanical dystocia by disproportion foeto-pelvian or anomaly of presentation.

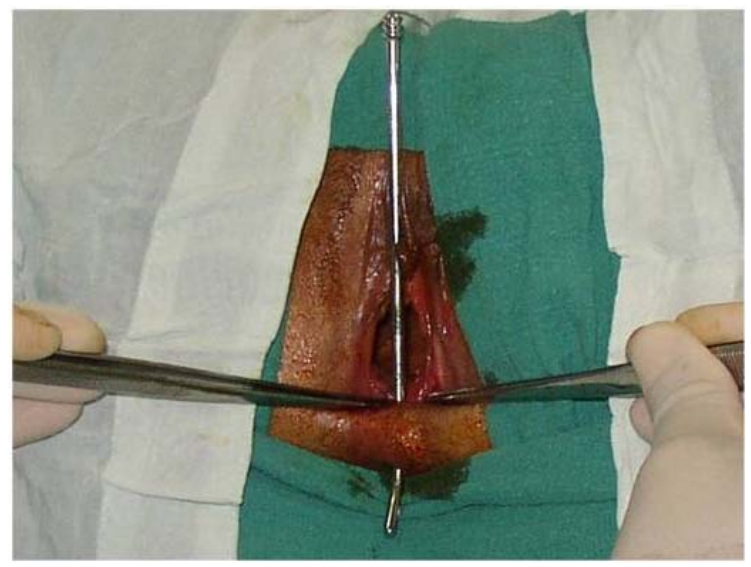

Figure 1. Fistulous route located by the stylet. 
- The vulvo-vaginal tissular anomalies by vaginal hypoplasy.

- The traumatic fistulas representing 10\% - 15\%. It can be of instrumental operations (pair of forceps) or surgical (caesarian).

Clinically, the most constant and the most revealing sign is the perception of the passage of gas and sometimes liquid stools by the vagina, and can associated to it or exist independently of the vaginal discharge more or less purulent, and of recurrent vaginal and/or urinary infections.

A complementary check-up has to comprise an endoanal echography to detect a possible sphincteric rupture, estimate the routes, the purulent cubicles and the associated perineal lesions.

Other examinations: MRI, ano-rectal manometry and defecography, biopsy are made under request [5-8].

Numerous classifications were proposed:

The classification proposed by Rosenshein in 1980 is a recognized authority $[9,10]$. It distinguishes the fistulas of the inferior third with destruction of the perineal body (type A) or with conservation of the perineal body (type $\mathrm{B})$, the fistulas of the average third (type C) and the fistulas of the superior third (type D). The first three types represent by themselves about $90 \%$ of the set.

On the practical plan, Rothenberger presented in 1983 $[11,12]$ :

- The simple fistulas: the inferior or average third of the vagina diameter is inferior or equals $2.5 \mathrm{~cm}$ banal traumatic or infectious cause.

- The complex fistulas: superior third of the vagina. Diameter superior to $2.5 \mathrm{~cm}$ Crohn, $\mathrm{RCH}$, irradiated tissues, cancers cause.

Numerous treatments were proposed and the surgical techniques are multiple [11]:

- CaeserianTechniques: They concern certain complex fistulas top situated. The suture with interposition of big epiploon finds its indication in the highly placed fistulas and in this case, the protection by a stoma is the rule.

- Vaginal way Technique: They contain the repair after fistulotomy and sphincter rice section (Musset's episio-proctotomy), the direct sutures of the fistulous opening by perineo-vaginal access or by trans-anal access, the injection of biological glue and the transpositions of autologous tissues be it about the gracilis muscle or about the bulbo-cavernous muscle.

- Direct suture after perineal or perineo-vaginal access by realizing a section at the level of the transverse fork or slightly arciform towards back, closer of the vagina than of the anus. The surgeon has two options: either he pursues in the wall until the fistulous route thus pure perineal way, or he re-splits vertically the posterior wall of the vagina of the fork up to the secondary fistulous opening using then a perineo-vaginal way. The vaginal opening can be treated within two manners, either a pure perineal or a perineo-vaginal approach where the vaginal opening is excised and the vagina is closed on almost all its height.

- Direct suture after trans-anal access with better exposure, the patient is in ventral decubitus.

- Technique of Musset is usually realized in 2 separated periods: from 6 to 12 weeks. The first period is a median perineotomy, which allows a view-to-view of the fistulous route. The second period is realized on a healed and clean perineum. It contains a repairing of the anal canal, a sphincteric repairing associated with the anterior myorraphy of levators ani and a vulvovaginal reconstruction. This technique gives excellent results and seems indicated when there are sphincteric lesions associated with the fistula.

- Technique flap of rectal reduction: the purpose is to realize a detachment of the entire rectal wall on an anterior hemi-circumference rising until approximately $8 \mathrm{~cm}$ of the pectinate line. Incision begins in 1 $\mathrm{cm}$ under the rectal opening located by a stilet, on the anterior hemi-circumference and the dissection continues in the septum. The flap is thus trimmed at the expense of all the wall of the rectum.

- Technique flap of vaginal reduction with a transversal incision at the expense of the posterior vaginal wall on all its width and starts at $1 \mathrm{~cm}$ under the secondary opening.

- Techniques of muscular interposition can give good results [8,9]: Numerous processes of transposition were described. In practice, only the transpositions from gracilis and bulbo-cavernous muscles are used because they are very effective for decay.

The indications of these various techniques take into account several factors:

- The breech of the fistula: Size, length and the number of routes, existence or absence intermediary diapyesis, mechanism of occurrence, etiology, quality of tissues, experience and school of the surgeon.

Recto-vaginal fistulas must not be operated before the fistulous opening is hemmed. It is recommended to wait 2 to 3 months and the operation must be preceded by an intestinal preparation [13].

The rates of success in the treatment of the simple fistulas are almost $100 \%$ and the treatment of the complex fistulas is exposed to a rate of failure which varies between $20 \%$ and $50 \%$ according to the statistics [13].

In case of vaginal delivery, the fistula reappears in more than $30 \%$ of the cases and $10 \%$ in case of urgent caesarian hence the necessity of realizing a scheduled systematic caesarian surgery. 


\section{CONCLUSION}

The recto-vaginal fistulas are frequent and of very varied etiologies. The quality of life of the patients is always much altered and the psychological, family and socioprofessional repercussions are major. Sometimes, their difficult treatment depends on multiple factors, which it will be necessary to estimate before any therapeutic approach.

\section{REFERENCES}

[1] Badenoch, D.F., Tiptaft, R.C., Thakar, D.R., Fowler, C.G. and Blandy, J.P. (1987) Early repair of accidental injury to the ureter or bladder following gynaecological surgery. British Journal of Urology, 59, 516-518. doi:10.1111/j.1464-410X.1987.tb04866.X

[2] Benchekroun, A. (1992) Hydraulic valve for continence and antireflux. A 17-year experience of 210 cases. Scandinavian Journal of Urology and Nephrology, 142, 66-70.

[3] Benchekroun, A., Lakrissa, A., Essakalli, H.N., Faik, M., Abakka, T., Hachimi, M., et al. (1987) Vesico-vaginal fistula about 600 cases. Journal of Urology, 93, 151-158.

[4] Ockrim, J.L., Greenwell, T.J., Foley, C.L., Wood, D.N. and Shah, P.J. (2009) A tertiary experience of vesicovaginal and urethro-vaginal fistula repair: Factors predicting success. BJU International, 103, 1122-1126. doi:10.1111/j.1464-410X.2008.08237.x

[5] Emembolu, J. (1992) The obstetric fistula: Factors associated with improved pregnancy outcome after a success- ful repair. International Journal of Gynecology \& Obstetrics, 39, 205-212. doi:10.1016/0020-7292(92)90658-6

[6] Evoh, N.J. and Akinla, O. (1978) Reproductive performance after the repair of obstetric vesico-vaginal fistulae. Annals of Clinical Research, 10, 303-306.

[7] Falandry, L. (1992) Urethro-vagino-fistula: Diagnostic and surgical techniques. Journal of Surgery, 129, 309-316.

[8] Rosenshen, N.B., Genadry, R.R. and Woodruff, D. (1980) An anatomic classification of rectovaginal septal defects. American Journal of Obstetrics and Gynecology, 137, 439-442.

[9] Rothenberger, D.A. and Goldberg, S.M. (1983) The management of recto-vaginal fistulae. Surgical Clinics of North America, 63, 61-79.

[10] Miklos, J.R. and Kohli, N. (1999) Rectovaginal fistula repair utilizing a cadaveric dermal allograft. International Urogynecology Journal, 10, 405-406. doi:10.1007/s001920050069

[11] Dargent, D. (1995) Thierry Adam, Patrice Mathevet Fistules rectovaginales. EMC, 41-870.

[12] Safan, A., Shaker, H., Abdelaal, A., Mourad, M.S. and Albaz, M. (2009) Fibrin glue versus martius flap interpositioning in the repair of complicated obstetric vesicovaginal fistula. A prospective multi-institution randomized trial. Neurourology and Urodynamics, 28, 438-441. doi:10.1002/nau.20754

[13] De Ridder, D. (2009) Vesicovaginal fistula: A major healthcare problem. Current Opinion in Urology, 19, 358361. doi:10.1097/MOU.0b013e32832ae1b7 